\title{
Educational workload and its psycho- physiological impact on student organism
}

\author{
Oleksandr Burov ${ }^{\mathrm{a},},{ }^{*}$, Oleksandra Tsarik ${ }^{\mathrm{b}}$ \\ ${ }^{a}$ Institute of Gifted Child, 11 a Salutna Str., 03190, Kiev, Ukraine \\ ${ }^{\mathrm{b}}$ National Aviation University, 1 Komarova Ave., Kiev, Ukraine
}

\begin{abstract}
It is described the method to study students' stability to perform learning tasks under impact of internal and external factors that includes special tests of performance (cognitive, perceptual, strength and mobility of neural processes, selfassessment, questionnaires) and measurement of physiological parameters in parallel (EEG and/or REG, heart rate, blood pressure). It was confirmed high self-descriptiveness of fluctuation structure of test tasks performance in relation to the physiological "cost" of performance and subjective assessment of mood and capacity. It was revealed a high relationship both rate and accuracy indices of test performance with parameters of speed and density of solar wind that has individual dependence on lag between those parameters with time and had more significant meaning comparing to over-week education workload.
\end{abstract}

Keywords: psychophysiology, external factors, measurement, learning, performance

\section{Introduction}

The possibilities of psychophysiological researches of a mental work have a high potential both methodologically, and methodically. Over the last decade the new methods were developed which have shown a high enough exactitude of the prognosis of a human state and serviceability when performing an operator work. In most cases they are directed on an assessment of human psychological possibilities. This corresponds to idea of a central role of cognitive processes in prediction of training and operation [1]. The positive correlations of psychophysiological and professional indices of operation demonstrate that the common cognitions always represent itself as the factor of the higher order. It is necessary to give preference to the tests based on an estimation of cognitive components, chronometric methods, estimation of a velocity of nervous processes in the future estimations of ability of the pilot or other operator controlling a complicated engineering system.

On the other hand, it is known that the oscillations of psychophysiological indices of a human state and serviceability have an exogenous and endogenous nature, and their phases are affected by a wide spectrum of rhythms, which are divided on ultradian, infradian, circadian [2] and high-frequency rhythms of physiological functions [3 etc.]. The periodic oscillations 10-18 days are detected in a functional state of the nervous and muscular system, plastic and power metabolism (including, oxidizing processes). Such modifications explain variable (oscillatory) character of a human serviceability, their professionally important psychophysiological qualities. However the latter rhythmic structure, the quantitative performances of their parameters are not always taken into account (especially as a system) during psychophysiological researches because of insufficient studying of oscillatory character of cognitive processes and processes of perception of the information by a human, on which the mental activity is created.

As a whole, the definition of serviceability of an operator is not precise in modern comprehension, and taking into account the requirements variation to separate (specialized) professional groups of the experts, demands also various criteria. In view of this, the estimation of serviceability of an operator is expedient for building outgoing not of common representations about a state of health of the man, and

\footnotetext{
*Corresponding author. E-mail: o.burov@iod.gov.ua.
} 
from a relation (balance) of physical and mental professionally important qualities. Accordingly, the integral estimation of serviceability should be under construction in view of specificity of a professional quota and with the registration infradian and ultradian rhythms, and also external including heliophysic factors when carrying out medico-biological and psychological inspection of operating staff.

\section{Method}

3 groups of 20 subjects participated (males volunteers aged 18-50 years) take part in 6 experiments separated by 1 week \pm 1 day to take into account ultradian rhythms influence ( $30 \pm 10$ days) on individual and common psychophysiological peculiarities of mental work, but excluding a week rhythm influence.

The effectiveness of research psychophysiological techniques considerably rises in case of usage not of a set of tests, but of a psychodiagnostic system. To solve this task the computer system of psychophysiological researches of a human psychomotor and cognitive activity in conditions of research laboratories and rehabilitation centres was developed [4]. Experiments included psychological tests performance by subjects at the computer display and simultaneous measurement of physiological parameters. They were used tests as follows: short memory, perceptual (searching of missed numeral), cognitive (logic-combinatorial). In all tests we registered time of each task performance in milliseconds, correct (expected) and really entered answers. Besides, we used a subjective state assessment of the examinees by means of the reduced variant of the test "General state - Activity - Mood" (GsAM) at the beginning and at the ending of the test session. (the indices of mood mood, serviceability FfD, attention atten, anxiety anxiety) prior to the beginning (index " $\ll 0 "$ ) estimated and upon finishing the tests performance.

As indices of physiological "cost" of activity and the human state we registered a heart rate HR and blood pressure (systolic ADs, diastolic ADd) by means of the cardiomonitor "Solveig". The indices HR, ADs and ADd we registered during $10 \mathrm{~min}$ prior to the tests beginning (index " 0 ") and $10 \mathrm{~min}$ after finishing (relaxation), as well as every 20 min during the test activity. The experiments differed by the test workload: training E1 (60 min); variable test workload E2 (160 min); constant load (160 min): E3, E4 and E6 - fixed rate calculated as average by results of the appropriate test performance in E2, E5 - free rate ("«auto-pace"). 15 volunteers participated in this series of experiments. Other group of the volunteers of 24 men fulfilled the same tests, but the rate was opposite: in E3, E4 and E6 - free rate, in E5 - fixed.

The specific of the research technique consisted in to check the variability in time of psychophysiological indices registered when subjects performed cognitive and perceptual tests, under impact of infradian rhythms of a various origin. With this purpose the schedule of research was developed that provides for involvement of the volunteers (males of 18-40 year old) in experiments with an interval 1 week \pm 1 day to take into account the influence of infradian rhythms (30 \pm 10 days) on psychophysiological $(\mathrm{PPh})$ regulation peculiarities of mental activity, but with elimination of influence of week rhythms. Each examinee took part in experiments with constant workload in the same phase of day to eliminate the circadian effect.

The data on influence of solar activity on a human health and some physiological systems are known, however results of study of cognitive activity associating with heliophysic parameters are not known in the scientific literature to date. In our preliminary pilot researches the precise connection between effectiveness of operator activity and parameters of a solar wind (SW) was revealed. With the purpose to study this phenomenon we registered indices of proton component of a solar wind - velocity SWsp $(\mathrm{km} / \mathrm{s})$ and density SWden (proton $/ \mathrm{sm}^{3}$ ) on the data from Internet site NASA [5], as well as parameters of the geomagnetic field (GMF) - planetary index Ks and index of "equivalent amplitude" $\mathbf{A}$.

\section{Results}

Usually in research of operator serviceability it is accepted that it forms under influence of a set of psychological $\Psi$ and physical $\varphi$ parameters of a human state. It is possible to estimate and to calculate operator serviceability (OS) as an integral index of his efficiency [6]. However for practical purposes estimations of OS often are more expedient for using an estimation of a velocity of a decision making at realization of cognitive tasks on the assumption of the given minimum level of reliability $\eta$. In empirical researches it was revealed that for the normal motivated activity and lack of stress in average $\boldsymbol{\eta}=0.95$. Taking into account probable $\sigma$ deviations for a group of operators it was accepted as admissible 
$\eta \geq 0.92$. In this case OS can be estimated by means of biorhythmic structure of time performance of cognitive tasks. Thus the accuracy of the prognosis by the model using such indices makes up the average 85$90 \%$.

To check possibilities of the created technique of psychophysiological researches the analysis of the obtained data was carried out in such directions variation of psychophysiological indices, correlation of psychophysiological parameters with external conditions.

The analysis of psychophysiological indices variation on days of the same experiments (E3, E4 and E6) specifies a high sensitivity of a velocity of the test tasks performance in the cognitive test simultaneously with variation of the solar wind parameters. The indices of a subjective estimation of mood and OS are rather stable during one month in contrast to the index of anxiety. At the same time data in experiments without time deficiency (E5) essentially differ from other experiments (Table.1). The mean tasks performance time $\mathbf{T l}$ in this experiment was authentic slower and reliability $\boldsymbol{\eta}$ higher. Accordingly inter-individual variation of $\mathbf{T l}$ reached $40 \%$, while $\boldsymbol{\eta}$ practically was absent $(1 \%)$ that is natural, because the volunteers were not exposed to "time pressure".

Among 6 experiments carried out by all subjects they were obtained data valid data in experiments E3 and E4 (free tempo over 3 hours of the same type tasks performance) that allow to analyze the physiological "cost" of the cognitive test performance. Physiological indices were measured and averaged over 3 phases - beginning, middle and at the end of the experiment. It was carried out the correlation analysis for indices of physiological maintenance of mental work in the experiment E3 with the indices of intelligence structure calculated by the result of the R.Amthauer's test ( 8 subtests) and the subjective test "General_state - Activity - Mood" as well as they were analyzed relationships between physiological indices.

The highest relationship between one of the physiological indices is a relationship between inter-beat intervals' spectrum density with sub-tests $\mathrm{AN}$ and RA as well as non-verbal intellect. It is necessary to note that the value of the relationship factor is the same for both low-frequency and high-frequency bands, but the sign of correlation is contrary. In other words the total energetic "cost" of test performance is relatively the same for both bands, and increase of low-frequency fluctuations is accompanied by decrease of high-frequency ones and vise versa.
High negative values of the relationship factor between blood pressure systolic with values of sub-tests RA and ZR $(r=-0,99)$ could be explained by that high mental abilities to do mathematical calculation tasks need low strain of cardio-vascular system while cognitive test (logical-and-combinatorial) performance. Index of blood pressure lateral has the high value of the relationship factor with the same indices (abilities) as well (correspondingly, $\mathrm{r}=0,93$ and 1,0 ). However, these sub-tests as sub-test FS in relation to blood pressure systolic $(\mathrm{r}=1,0)$, conversely, need high energetic efforts that is demonstrated by the negative sigh of correlation.

In general, it is necessary to highlight that the most energetically "cost-expensive" is a sub-test RA, i.e. it requires some additional effort from the cardiovascular system, because its performance has a high relationship factor and with other physiological parameters as well. F.e., Bayevsky index of myocardium strain has a high relationship only with this subtest $(\mathrm{r}=0,85)$ that corresponds the idea concerned a relationship between mental work output and its physiological maintenance. It is necessary to add that Bayevsky strain index demonstrate a high relationship and with blood pressure lateral $(\mathrm{r}=-0,99)$ and blood pressure systolic $(r=-0,99)$ that could be explained by relationship of baroreflex with mental performance exertion. But this needs additional investigation, because the cognitive test was performed in this experiment in free tempo and nervousemotional strain appears only as subject to a high motivation to the particular activity if a time "pressure" is absent.

The data have shown that the subjective estimations of the examinees and heliophysic indices also essentially differ in days of experimental researches, i.e. both psychological, and the external physical factors are not stable conditions of research carrying out. However, it is paid attention to improving of tests results in experiments E4 and E6, and an increase of a velocity of the solar wind on the same days. The relationship between these groups of indices was studied by means the factor analysis with use of varimax rotation of principal components.

Among analyzing variables, 4 factors are chosen which are clear divided by factor loadings. So, in the first factor the greatest contribution is made by indices of a subjective estimation of a state, reliable characters of operation in the test, diastolic pressure before the beginning of testing and SW speed. Thus, this factor is shaped by the initial state of the human and his subsequent reliability under influence of the SW proton component. 
The second factor is created, mainly, by the SW density and variation of parameters of the cardiovascular system during adapting an organism to conditions of operator activity (first 20 minutes in 3-hour researches). It is interested the association namely of physiological reorganizations, instead of indices of activity, with given the heliophysic parameter, in contrast with the $3^{\mathrm{d}}$ factor that is created by indices GMF and rate of cognitive tasks performance (mean value, and its variation).

The values of psychophysiological parameters of cognitive activity and subjective estimations of a human state depend not only on character of the tasks fulfilled, but also on a phase of infradian rhythms that should be took into account when carrying out psychophysiological researches. Intra-individual variation of most psychophysiological indices could very from 10 to $38 \%$ over four consequent weeks.

The further study of relationship of a human activity and heliophysic influences is carried out by means of multiple regression analysis. We studied relationship of indices of the test tasks performance and subjective estimations (TTSE) with indices of physiological maintenance (the serial number "i" corresponds consecutive $20 \mathrm{~min}$ of research), SW and GMF. As the partial correlations between parameters considerably differ both as a whole, and in each experiment, more important, from the point of view of an estimation and prediction, is the coefficient of multiple correlation $\mathrm{R}$ of each single parameter of activity and mood with the most informative parameters of environment and physiological maintenance. We carried out the selection of such parameters by means of stepwise regression analysis with usage of the package of statistical programs STATISTICA v5.5 (selection results are marked by “+” in the table 3 for each parameter TTSE). The values of multiple correlation factor $R$ and level of reliability $p$ for the appropriate equations of regression are pointed.

It attracts attention also that dependence of tasks performance pace indices on parameters of an external environment disappears. It is obvious, that in conditions of lack of time deficiency as a sort of stress the psychophysiological self-regulation ensures sufficient compensating of external influences on integral activity of a human. At the same time, reliability of operation and the subjective estimations of a state remain dependent on heliophysic factors that can have an effect for behavior of a human and his interactions with other persons. Probably, the indicated parameters influence on cognitive aim and as a result - on effectiveness of operator work [7].

Different psychological types of subjects have demonstrated non-equal response to external impact. The most stable type were introverts, and extraverts demonstrated more rapid, but not-stable work under conditions that have changed.

It is proposed to keep in mind for school teachers, as well as managers that a human cognitive abilities depend not only on skills and motivation, but objective external factors that usually are out-of-attention regards particular student (worker). Organizational efforts are needed to compensate a gap between a human fitness-to-work and work conditions.

\section{References}

[1] Carretta, T.R.; Ree, M.J. U.S. Air Force Pilot Selection Tests: What Is Measured and What Is Predictive? Aviation, Space, and Environmental Medicine. 1996, V. 67, 3. 279-283.

[2] Halberg, F., Halberg, E., Barnum, C. P., and Bittner, J. J. (1959). Physiologic 24-h periodicity in human beings and mice, the lighting regimen and daily routine. In $\mathrm{R}$. B. Withrow (Ed)., Photoperiodism and related phenomena in plants and animals (Vol. 55, pp. 803-878). Washington: American Assoc. for the Advances in Science.

[3] Baevski R.M. Prediction of states on the verge of norm and pathology. M.: Medicine, 1979. - 318.

[4] Automation of experiment for psychophysiological research in work physiology. Решетюк А.Л., Burov O.Yu, Polyzkov O.A. et al. - MOH of Ukraine, Kiev, 1993. 11.

[5] SEC's Anonymous FTP Server (Solar-Geophysical Data) < http://sec.noaa.gov/ftpmenu/lists/ace2.html $>$

[6] Polyakov A.A., Burov A.Yu., Korobeinikov G.V. Functional organization of mental work of people of different. Phiziol. Chelov. 1995, V.21, № 2.- 37-43.

[7] Kostandov E.A. Psychophysiology of consciousness and unconscious. - S.-Pt.: Piter, 2004. 167. 